\title{
A busca por violações da simetria de Lorentz: testando os princípios da relatividade restrita na escala de Planck
}

The search for violations of the Lorentz symmetry: testing the principles of special relativity in the Planck scale

\author{
A. F. Ferrari ${ }^{*}[0$ \\ ${ }^{1}$ Universidade Federal do ABC, Centro de Ciências Naturais e Humanas, Santo André, SP, Brasil
}

Recebido em 22 de Abril, 2019. Aceito em 13 de Maio, 2019.

\begin{abstract}
A relatividade restrita, proposta por Einstein no começo do século XX, veio a se tornar um dos pilares da física. Contudo, uma das lições que aprendemos com ela é que teorias físicas geralmente tem seu domínio de validade: uma teoria de grande sucesso em descrever certos fenômenos pode simplesmente deixar de valer se extrapolada para um novo regime. Desta forma, a teoria da relatividade também pode ter seu domínio de validade, e é fundamental testar seus princípios em regimes que não foram ainda investigados. Um exemplo é na chamada escala de Planck, onde a compreensão atual da mecânica quântica e da gravitação mostra-se incompleta, e justamente por isso esperamos o surgimento de nova física. Vamos discutir como a busca por violações da simetria de Lorentz, o princípio fundamental da teoria da relatividade restrita, se apresenta como uma janela para a investigação da física na escala de Planck. Boa parte desse trabalho pode ser lido como um texto de divulgação científica, mas ao final apresentamos uma análise da eletrodinâmica com violações de Lorentz, pensada para leitores com uma formação de física em nível de graduação.
\end{abstract}

Palavras-chave: Violações de Lorentz, Teoria da Relatividade, Gravitação Quântica, Eletromagnetismo.

Special relativity, proposed by Einstein at the beginning of the twentieth century, has become one of the pillars of physics. However, one of the lessons we have learned from it is that physical theories generally have their domain of validity: a highly successful theory describing certain phenomena may simply stop working if extrapolated to a new regime. Therefore, relativity theory may also have its validity domain, and it is fundamental to test its principles in regimes that have not yet been investigated. An example is the so-called Planck scale, where the current understanding of quantum mechanics and gravitation is incomplete, and precisely because of that, we expect the emergence of new physics. We will discuss how the search for violations of Lorentz symmetry, the fundamental principle of the theory of special relativity, presents itself as a window to the investigation of physics in the Planck scale. Much of this work can be read as a text of science popularization, but at the end we present an analysis of the electrodynamics with Lorentz violations, intended for readers with a knowledge of physics at undergraduate level.

Keywords: Lorentz violations, Relativity Theory, Quantum Gravity, Electromagnetism.

\section{Introdução}

Albert Einstein foi uma das figuras mais influentes da física no século XX. Além de suas enormes contribuições ao avanço do conhecimento científico, Einstein ganhou notoriedade junto ao público pela ocasião das expedições que se utilizaram de um eclipse solar ocorrido em 1919 para verificar seus cálculos acerca da deflexão da luz pelo Sol - uma das expedições, justamente a que realizou as melhores medidas, veio a Sobral, no Ceará, realizar suas observações [1 3. Esse marco da física do século XX e da física no Brasil em particular comemora seu primeiro centenário neste ano de 2019, e por isso podemos dizer que há um século Einstein tem sido, para muitos, a personificação da genialidade. Com a notorie-

*Endereço de correspondência: alysson.ferrari@ufabc.edu.br dade, veio também a atenção de curiosos e entusiastas, que julgaram encontrar defeitos ou inconsistências óbvias em suas teorias - muitas vezes movidos pelo entusiasmo de "revolucionar a física" a partir de raciocínios simples e diretos, justamente como o próprio desenvolvimento da relatividade é muitas vezes descrito em livros de popularização da ciência. Sabemos contudo que a teoria da relatividade não possui nenhuma inconsistência óbvia. Mais que isso, ao longo do século XX ela acumulou sucesso tão extraordinário, não somente nas suas verificações experimentais, como também no seu papel como um dos pilares da descrição teórica de todas as interações fundamentais conhecidas, que a idéia de buscar novas confirmações experimentais dos seus princípios básicos pode parecer desnecessária. Contudo, vamos argumentar que essa busca não é apenas válida, como pode ser uma potencial janela para observação de uma nova fí- 
sica, associada à gravitação quântica. Em particular, a abordagem que iremos descrever busca por violações da simetria de Lorentz, que, como veremos, é o princípio fundamental a partir do qual toda a relatividade restrita é construída. A ferramenta básica para essa busca é o Modelo Padrão Estendido desenvolvido por Kostelecky e colaboradores 4,5], que tem servido de guia para uma busca sistemática por violações da simetria de Lorentz através de diferentes técnicas experimentais.

Procurando por possíveis efeitos que estejam em desacordo com os princípios da relatividade restrita, podemos descobrir evidências de novos fenômenos físicos, e talvez iniciar uma revolução na compreensão do espaço e do tempo. Por outro lado, a confirmação da validade da simetria de Lorentz, mesmo em escalas de comprimento extraordinariamente pequenas, onde temos razões para acreditar que uma nova física deve existir, seria também uma informação científica de grande relevância. Até o presente momento, esse segundo cenário é o que tem se concretizado, como resultado desses estudos: a busca por violações de Lorentz tem gerado um conjunto impressionante de novas verificações experimentais, extremamente precisas e não triviais, da relatividade restrita.

Ao escrever este trabalho, tentou-se alcançar um público com interesse pelo tipo de questão esboçada nesta introdução, e que possua ao menos um certo conhecimento da física do século XX conforme exposta em livros e revistas de popularização de ciência. As seções 2, 3 e 4 elaboram as principais motivações para se procurar por possíveis violações da simetria de Lorentz e são escritas com uso mínimo de equações. Essas seções em conjunto formam o que pode ser lido como um texto de divulgação científica, que busca trazer a um público mais amplo as principais motivações por trás dessas pesquisas. A seção 5 elabora algumas idéias mais específicas que norteiam como essa busca será desenvolvida, e alguns conceitos um pouco mais avançados de física são utilizados. Finalmente, na seção 6 supomos que o leitor tenha uma formação de física em nível universitário (essencialmente, eletromagnetismo e relatividade restrita, em nível de graduação), e apresentamos algumas das técnicas e resultados fundamentais da área, no contexto do eletromagnetismo com violações de Lorentz. Finalmente, algumas perspectivas e considerações finais são apresentadas na seção7.

\section{Os limites da Mecânica Newtoniana, e o surgimento da relatividade restrita}

O surgimento da mecânica Newtoniana no século XVII é um dos marcos daquilo que reconhecemos como a ciência moderna, e ao longo dos séculos seguintes, não apenas sua formulação foi progressivamente refinada (surgindo as formulações lagrangeanas e hamiltonianas da mecânica, por exemplo) como sua aplicação nos mais diversos fenômenos somou sucessos e comprovações experimentais. Talvez o mais claro exemplo de sucesso da mecânica de Newton, em conjunto com sua teoria de gravitação, foi a previsão da existência de novos planetas a partir das pequenas divergências entre os cálculos teóricos e as observações das órbitas planetárias, como no caso de Netuno em meados do século XIX. Naquele momento, dificilmente se poderia negar à mecânica de Newton o status de "verdade científica". Apesar disso, pouco mais que cinquenta anos depois destas confirmações extraordinárias das teorias Newtonianas, a comunidade física reconhece suas limitações e a validade de uma nova teoria que a substitui, modificando tanto seus conceitos básicos quanto suas equações fundamentais - a relatividade restrita. Aprendemos assim que, por mais bem testada experimentalmente, por mais logicamente consistente e mesmo esteticamente atraente, uma teoria física jamais pode ser tomada como uma verdade definitiva ou incontestável. "Verdades científicas", são questionáveis e devem ser embasadas, em última instância, num amplo conjunto de observações experimentais, assim como numa extensa investigação da sua consistência teórica.

O que acontece é que, em geral, teorias científicas tem um domínio de validade ${ }^{1}$ e fornecem uma descrição correta da natureza apenas para fenômenos dentro deste domínio. No final dos séculos XIX e começo do XX, o avanço das técnicas experimentais permitiu a exploração de novos fenômenos, que hoje pertencem ao domínio da física atômica e molecular, bem como da física relativística. Nesses novos domínios, a aplicação da mecânica Newtoniana, junto das outras teorias físicas então consideradas bem estabelecidas, levaram a paradoxos e mostraramse incapazes de descrever os fenômenos observados. A conclusão a que se chega é que a teoria de Newton não funciona nesses domínios - ela está "errada", mas da uma forma desafiadora do senso comum e do uso cotidiano da palavra "errada": ela funciona muito bem num determinado conjunto de fenômenos, mas deixa de ser uma boa descrição para fenômenos fora deste domínio, devendo ser substituída por outra teoria mais "completa". É curioso que essa teoria mais "completa" pode envolver conceitos que são radicalmente diferentes da teoria inicial - como exemplificado dramaticamente no caso da mecânica quântica - e, no entanto, a teoria "errada" pode sempre ser de alguma forma reconhecida como um caso limite da nova teoria, continuando a ser muitas vezes a descrição mais conveniente da natureza dentro do seu próprio domínio de validade. Justamente por isso a mecânica Newtoniana continua sendo importante, tanto na prática, quanto para investigações teóricas, mesmo após o surgimento da mecânica quântica e da relatividade. "Verdades científicas", enfim, são geralmente aplicáveis a um certo domínio de

\footnotetext{
${ }^{1} \mathrm{O}$ que queremos nos referir como um "domínio" é um conjunto de fenômenos, geralmente identificado pela ordem de grandeza típica das características físicas relevantes (massas, cargas, dimensões, etc...) das entidades que participam desses fenômenos. Assim, por exemplo, o "domínio" da mecânica clássica se refere a fenômenos envolvendo corpos de dimensões e massas muito maiores que às associadas a átomos, e velocidades muito menores que a velocidade da luz.
} 
fenômenos, onde elas de fato podem ser adotadas como "verdadeiras" para todos os fins práticos: aplicadas fora deste domínio, podem ser completamente incompatíveis com o que se observa na natureza.

Aprendemos, também, que a identificação e exploração de novos domínios de fenômenos é um campo fértil para a descoberta de nova física. No final do século XIX, um conjunto bem estabelecido de teorias - a mecânica, o eletromagnetismo e a termodinâmica - vinha sendo sistematicamente, e com grande sucesso, aplicado a uma vasta gama de experimentos e observações. Chegou-se até a uma sensação de que o "fim da física" estava próximo: podemos lembrar aqui a afirmação de Lord Kelvin acerca das "nuvens" que perturbavam o céu de brigadeiro da física clássica 6]. Contudo, uma vez que técnicas experimentais foram desenvolvidas permitindo o estudo do comportamento de pequeno número de moléculas e átomos - abrindo a porta ao mundo quântico - e que outras técnicas chegaram a precisão suficiente para medir efeitos suprimidos pela razão entre velocidades de certos sistemas físicos e a velocidade da luz - explorando efeitos relativísticos - uma sucessão impressionante de descobertas marcou o começo do século XX. Teorias físicas consolidadas foram colocadas à prova, e o resultado foi uma revolução na física, em que ao longo de duas décadas, a teoria da relatividade e a mecânica quântica se estabeleceram como novos pilares da física. Uma "verdade científica", estabelecida com a consolidação de uma teoria científica descrevendo um certo conjunto de fenômenos, não necessariamente demarca o fim de um ramo da ciência, mas delimita onde podemos procurar novas e interessantes questões.

\section{O problema da gravitação quântica e os limites da física fundamental do século XXI}

O século XX presenciou o surgimento e a maturação de várias das peças fundamentais da nossa atual compreensão do universo: a Relatividade Restrita - estabelecendo a noção do espaço-tempo como a arena onde a física se desenvolve -, a Relatividade Geral - tornando o espaço-tempo uma entidade dinâmica, englobando em si mesmo, de forma geométrica, a gravitação -, e a Mecânica Quântica - que descreve o comportamento do mundo sub-atômico, de moléculas e átomos. Como iremos nos referir a essas teorias muitas vezes, definimos os seguintes acrônimos para elas: RR, RG e MQ.

A evolução desse conjunto de teorias se deu em duas frentes principais, tomando como base a RR. Por um lado, a RG estende a RR, descrevendo de forma clássica os fenômenos de origem gravitacional, mostrando-se em particular adequada para a descrição do universo nas grandes escalas de comprimento - culminando com a possibilidade de descrever a evolução do universo como um todo, com os modelos cosmológicos que tem sido formulados e testados por meio de observações astrofí- sicas com grande precisão 7,8]. Por outro lado, a MQ pode ser compatibilizada com os princípios da RR, através do que conhecemos como teoria quântica de campos (TQC), e fornece ferramentas adequadas para descrever as interações fundamentais entre as partículas elementares, com exceção da gravitacional. Este desenvolvimento culmina na formulação do Modelo Padrão (MP) das partículas elementares, que previu teoricamente a existência do bóson de Higgs, descoberto em 2012 no LHC [9 11]. Temos assim dois ramos da física que, essencialmente independentes, alcançam enorme sucesso na descrição de fenômenos distintos: a RG descreve a física de grandes escalas de comprimento, o domínio das estrelas, galáxias e do universo, onde a gravidade é a única interação que exerce papel relevante, e o MP descreve a física das pequenas escalas de comprimento, o domínio das partículas elementares, unificando num formalismo quântico três interações fundamentais (interação eletromagnética, nuclear fraca e forte), mas deixando de lado a gravitacional, que é tipicamente de intensidade desprezível nestas escalas de comprimento.

Tentativas de incorporar a interação gravitacional ao MP falham por uma série de dificuldades técnicas que até hoje não foram devidamente superadas, e surge daí o chamado problema da gravitação quântica: os métodos conhecidos para a quantização de uma teoria fundamental aparentemente não funcionam para a $\mathrm{RG}$, para a qual somente conhecemos uma formulação clássica 12. Esta aparente incompatibilidade entre duas descrições bastante distintas para interações fundamentais - a RG, geométrica, não-quantizada, valendo no domínio "macro", e o MP, quântico, valendo no domínio "micro" representa uma situação em que temos descrições incompatíveis para domínios de fenômenos. Esta situação é geralmente considerada insatisfatória, mas à primeira vista, poderíamos pensar que não representa em si um problema grave, já que cada um dos fenômenos que conseguimos explorar experimentalmente acaba se enquadrando em um ou outro destes domínios, e portanto pode ser descrito ou pela RG, ou pelo MP.

É defensável uma visão pragmática, segundo a qual essa aparente incompatibilidade entre a RG e o MP é uma idiossincrasia do universo em que vivemos, e não um problema científico a ser atacado? Acreditamos que não, porque se tivéssemos acesso a fenômenos numa intersecção entre esses dois mundos, então efetivamente não teríamos uma descrição teórica consistente para esses fenômenos, e estaríamos numa crise tão aguda como aquela que se viu no começo do século XX, quando ficou evidente a incompatibilidade entre a aplicação das idéias clássicas da física ao domínio atômico e relativístico. E de fato há pelo menos dois exemplos na natureza de sistemas onde os domínios da RG e do MP se sobrepõem: buracos negros, e o estado inicial do universo, segundo o "modelo cosmológico padrão" desenvolvidos nas últimas décadas. São fenômenos que não estão ao alcance de laboratórios terrestres (note contudo os interessantes de- 
senvolvimentos envolvendo simulações hidrodinâmicas de buracos negros 13 15]), e que certamente não seremos capazes de descrever em sua totalidade sem uma teoria de gravitação quântica. Podemos por exemplo comprovar a existência de buracos negros por observações astrofísicas, geralmente baseadas em propriedades puramente clássicas, e mesmo inferir alguns efeitos quânticos como a radiação de Hawking, mas ainda não possuímos uma compreensão completa destas estruturas com as ferramentas teóricas conhecidas no momento.

Um pouco de análise dimensional $[16$ ajuda a elucidar a razão para essa dificuldade de realizar experimentos que estejam na intersecção entre o domínio do "macro" e do "micro", ou seja, da RG e do MP, e para a distância enorme entre esses dois domínios da física. Sendo compatíveis com a relatividade restrita, tanto a $\mathrm{RG}$ quanto o MP envolvem a velocidade da luz

$$
c=299.792 .458 \mathrm{~m} / \mathrm{s}
$$

como uma constante fundamental. Por outro lado, na RG aparece também a constante da gravitação de Newton

$$
G \approx 6,67 \times 10^{-11} \mathrm{~N} \cdot \mathrm{kg}^{2} / \mathrm{m}^{2},
$$

que caracteriza a intensidade da interação gravitacional, ao passo que, na MQ, a constante de Planck

$$
\hbar \approx 1,05 \times 10^{-34} \mathrm{~J} \cdot \mathrm{s}
$$

controla a quantização das grandezas físicas. A partir destas três constantes universais, pode-se construir constantes com dimensão de comprimento, massa, energia, e tempo, denominadas comprimento de Planck

$$
\ell_{P}=\sqrt{\frac{\hbar G}{c^{3}}} \approx 1,61 \times 10^{-35} \mathrm{~m},
$$

massa de Planck,

$$
m_{P}=\sqrt{\frac{\hbar c}{G}} \approx 2,18 \times 10^{-8} \mathrm{~kg},
$$

energia de Planck,

$$
E_{P}=\sqrt{\frac{\hbar c^{5}}{G}} \approx 1,22 \times 10^{19} \mathrm{GeV},
$$

e tempo de Planck,

$$
t_{P}=\sqrt{\frac{\hbar G}{c^{5}}} \approx 5,39 \times 10^{-44} \mathrm{~s},
$$

onde adotamos o elétron-volt $e V \approx 1,60 \times 10^{-19} \mathrm{~J}$ como unidade de energia, o que é muito usual em física de partículas elementares. Essas definições levam ao sistema natural de unidades, amplamente utilizado na física teórica 16]. Além disso, essas definições tem um papel na delimitação do domínio da gravitação quântica - ou seja, dos fenômenos onde a incompatibilidade citada anteriormente se torna um problema fundamental.
Se nos perguntamos em que escala de comprimento a TQC se torna fundamental para o estudo de determinado fenômeno, podemos considerar o comprimento de Compton

$$
\ell_{C} \sim \frac{\hbar}{m c},
$$

que associa um comprimento de onda típico a um dado corpo de massa $m{ }^{2}$. Vamos agora fazer um tipo de raciocínio familiar para quem já fez um curso de MQ: se queremos investigar uma partícula de massa $m$ com uma precisão espacial da ordem $\ell_{C}$, necessitamos de fazê-la interagir com alguma partícula teste (que assumimos de massa nula). Trazendo da MQ a relação de incerteza de Heisenberg $\Delta x \Delta p \sim \hbar$ e da $\mathrm{RR}$ a relação entre energia $\mathrm{e}$ momento (para uma partícula sem massa ou altamente relativística), $E=c p$, podemos concluir que a energia dessa partícula teste será da ordem de $E \sim m c^{2}$, o que é suficiente para criar novas partículas de massa $m$. A conclusão é que fenômenos envolvendo partículas de massa $m$, em que são relevantes distâncias da ordem de seu comprimento de Compton $\ell_{C}$, podem envolver a criação/aniquilação de partículas, um efeito que é descrito por uma mecânica quântica relativística - que vem a ser, na sua formulação mais completa, a TQC. Em resumo: a $T Q C$ é fundamental em fenômenos associados a dimensões da ordem do comprimento de Compton dos objetos estudados.

Podemos também propor um argumento para encontrar uma escala de comprimento na qual efeitos gravitacionais precisam ser levados em conta, e a chave para isso é a noção de comprimento de Schwarzschild,

$$
\ell_{S} \sim \frac{G m}{c^{2}}
$$

sendo um dos resultados da RG que uma concentração de massa $m$ numa região de tamanho da ordem de $\ell_{S}$ leva à formação de um buraco negro - uma situação que envolve campos gravitacionais fortes, que certamente não podem ser descartados para uma correta descrição da física. Assim, a $R G$ é fundamental para fenômenos associados a dimensões da ordem do comprimento de Schwarzschild dos objetos estudados.

Agora, se procuramos por uma situação em que tanto efeitos da TQC quanto da RG sejam importantes, podemos imaginar que ela seja caracterizada por fenômenos envolvendo partículas de massa $m$ em uma certa escala de comprimento $\ell$ tal que $\ell_{C} \sim \ell_{S} \sim \ell$, ou seja, tanto $\ell_{C}$ quanto $\ell_{S}$ são compatíveis com a escala da física que estamos considerando. É um exercício interessante mostrar que $\ell_{C}=\ell_{S}$ justamente quando a massa considerada é a massa de Planck $m_{P}$ e, nesse caso, $\ell_{C}=\ell_{S}=\ell_{P}$. Ou seja, esperamos que efeitos quânticos de gravitação devem se tornar cruciais para o entendimento de fenômenos envolvendo partículas elementares de massa da ordem de $m_{P}$ e uma distância típica da ordem de $\ell_{P}$.

${ }^{2}$ Para objetos macroscópicos, $\ell_{C}$ é pequeno bastante para ser desprezível, e essa é uma forma de perceber que a mecânica quântica é irrelevante para a descrição de corpos macroscópicos. 
Note que $m_{P}$ é uma massa muito grande para uma partícula elementar, já que $m_{P} \sim 10^{-8} \mathrm{~kg}$ ao passo que por exemplo a massa de um próton é aproximadamente $10^{-27} \mathrm{~kg}$; da mesma forma, $\ell_{P}$ é uma escala de comprimento extremamente pequena, já que $\ell_{P} \sim 10^{-35} \mathrm{~m}$ enquanto que, por exemplo, o raio de um próton/neutron é da ordem de $10^{-16} \mathrm{~m}$, e o "microscópio" mais potente do mundo atualmente em operação, o próprio LHC, investiga escalas de comprimento da ordem de $10^{-19} \mathrm{~m}$.

O argumento acima certamente não é uma prova definitiva, já que não sabemos de fato se os princípios da física atualmente conhecida "funcionam" em um domínio de fenômenos tão distantes do que somos capazes de estudar. Apesar das limitações, contudo, esse argumento sugere que o comprimento de Planck $\ell_{P}$ pode ser tomado como um delimitador dos limites do nosso conhecimento da física, e do domínio onde uma nova física deve surgir - a física da gravitação quântica - papel similar cabendo às demais grandezas $m_{P}, E_{P}, t_{P}$. Do parágrafo anterior, o leitor percebe o quanto essas grandezas estão distantes das nossas capacidades experimentais atuais. Pensando em termos de comprimento, por exemplo, o maior acelerador de partículas do mundo explora fenômenos ocorrendo numa escala de comprimentos cerca de dezesseis ordens de magnitude acima de $\ell_{P}$. Isso significa, grosseiramente, que para o LHC a física da gravitação quântica está ainda tão distante quanto para um ser humano um próton está distante das suas possibilidades de manipulação direta, sem instrumentos (veja a Figura 1 .

Sempre podemos imaginar a construção de novos e maiores aceleradores de partículas, mas é difícil conceber, com a tecnologia atualmente conhecida, a construção de um acelerador que seja $10^{16}$ vezes mais poderoso que o LHC. Desta forma, é extremamente desafiador a realização de experimentos que investiguem a física da gravitação quântica. Apesar disso, ainda temos razões muito fortes para investigar esse problema. Em resumo: a situação atual da física fundamental, neste começo de século XXI, é de saber que muito provavelmente existe uma física nova e interessante na escala de Planck, que deli- mita os limites do domínio da física que hoje conhecemos; ao mesmo tempo, reconhecemos as enormes dificuldades de se fazer medidas e investigar diretamente fenômenos que se passem nesse domínio.

\section{Violações de Lorentz como uma janela para nova física na escala de Planck}

Existem muitas propostas para uma teoria de gravitação quântica, a mais famosa é a chamada teoria das cordas (string theory) [12], mas podemos citar também outras abordagens como gravitação quântica de laços (loop quantum gravity) 17, métodos computacionais como o das triangulações causais (causal dynamical triangulations) 1819 , geometria não-comutativa (noncommutative geometry) 20, etc. Vamos focalizar em apenas um aspecto comum a grande parte destas abordagens: a nossa compreensão do que é o espaço-tempo pode mudar radicalmente na gravitação quântica (uma bela discussão, no nível de divulgação cinetífica, ao que entendemos como espaço-tempo atualmente pode ser encontrada em [21]; uma introdução mais técnica, em [22]).

O argumento básico é: na RG, a gravitação está representada na própria geometria do espaço-tempo, através da sua curvatura. Uma gravitação quantizada, portanto, deve implicar em algum tipo de "quantização" do espaçotempq ${ }^{3}$ Essa idéia é uma das principais motivações para o que se chama de geometria não-comutativa, por exemplo [20]. Em várias abordagens como na quantização por laços ou triangulações causais, o espaço-tempo é substituído por uma estrutura "granular", onde esses "grânulos" tem tamanho típico dado por $\ell_{P}$. Já na teoria de cordas, o espaço-tempo é contínuo, mas sua dimensionalidade é bastante diferente do que observamos em baixas energias, correspondendo a 26 ou 10 dimensões

\footnotetext{
${ }^{3}$ Para quem conhece o formalismo da mecânica quântica: esse argumento faz um paralelo entre o espaço-tempo na RG e o espaço de fase, que é o cenário natural para se descrever a dinâmica de um sistema clássico, e que é "quantizado" na passagem para a mecânica quântica.
}
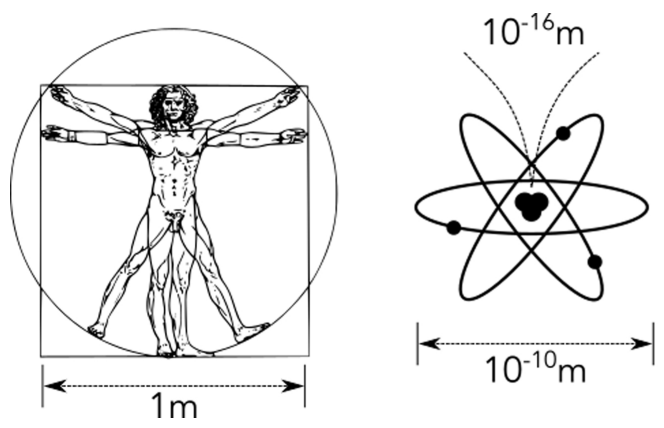

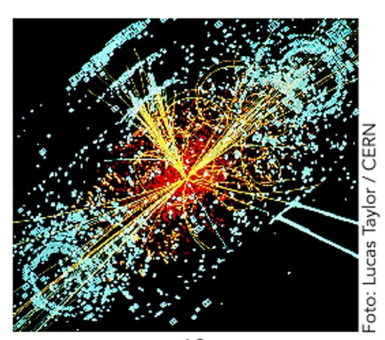

$10^{-19} \mathrm{~m}$

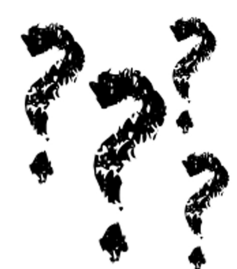

GRAVITAÇÃO QUÂNTICA $10^{-35} \mathrm{~m}$

Figura 1: Da esquerda para direita, o homem vitruviano de Leonardo da Vinci, uma representação simbólica do átomo, a reconstituição de uma colisão do LHC, e uma representação da "grande incógnita", a gravitação quântica, bem como a indicação das escalas de comprimento relevantes. Note que a diferença de tamanho entre o ser humano (da ordem de $1 \mathrm{~m})$ e o núcleo atômico $\left(10^{-16} \mathrm{~m}\right)$ é de 16 ordens de magnitude, que é similar à diferença entre a escala de comprimento associada às colisões do $\mathrm{LHC}\left(10^{-19} \mathrm{~m}\right)$ com a escala onde esperamos descobrir a gravitação quântica, que é $\ell_{P} \sim 10^{-35} \mathrm{~m}$. 
dependendo da versão da teoria de cordas, o que implica que boa parte das dimensões estão de fato "escondidas" de alguma forma - possivelmente, curvadas com um raio muito pequeno, em alguma forma geométrica que pode ser bastante complicada 12]. Em todas essas abordagens, o espaço-tempo apresenta ao menos algum tipo de desvio conceitual em relação à relatividade restrita.

Vamos refletir agora sobre as possíveis consequências do cenário aqui descrito para a relatividade restrita. Os fundamentos da RR estão intimamente ligados à geometria do espaço-tempo, através da noção de suas simetrias: em termos físicos, estamos nos referindo à liberdade de escolha de referenciais inerciais. É conhecido já, da mecânica de Newton, o fato das leis da física não mudarem sua forma frente a uma rotação ou uma translação da origem do referencial adotado, por exemplo. A RR extende essa noção incorporando as transformações entre referenciais que se movem um em relação ao outro com velocidade constante, mantendo a velocidade da luz a mesma para todos os observadores. Tipicamente chamadas de transformações de Lorentz em livros elementares [23], essas transformações fornecem a relação entre as coordenadas $(x, y, z)$ e tempo $t$ de um determinado evento conforme medidos por um referencial $S$, e as coordenadas $\left(x^{\prime}, y^{\prime}, z^{\prime}\right)$ e tempo $t^{\prime}$ do mesmo evento, medidos num referencial $S^{\prime}$ que se move em relação a $S$ com velocidade constante $v$ que, por simplicidade, assumimos seja na direção $x$. Com estas escolhas, as transformações de Lorentz podem ser escritas como

$$
x^{\prime}=\gamma(x-v t), t^{\prime}=\gamma\left(t-\frac{v x}{c^{2}}\right), y^{\prime}=y, z^{\prime}=z,
$$

onde

$$
\gamma=\frac{1}{\sqrt{1-v^{2} / c^{2}}} .
$$

Essas transformações trazem em si os aspectos mais revolucionários da RR, já que misturam tempo e espaço conforme medido por diferentes observadores. A contração dos comprimentos e a dilatação do tempo, famosos efeitos relativísticos, derivam diretamente dessas equações 23.

Neste ponto, contudo, temos que fazer um ajuste de nomenclatura: essas transformações são mais apropriadamente chamadas de boosts em inglês, algo como "empurrões" em português. Este último nome é mais adequado já que na literatura especializada se costuma chamar o conjunto tanto de rotações espaciais e de "empurrões" de transformações de Lorentz, e o fato de que as leis da física preservam sua forma frente a essas transformações significa que essa leis são invariantes frente à simetria de Lorentz. Passaremos a adotar essa nomenclatura de agora em diant $4^{4}$ A simetria de Lorentz pode ser enten-

\footnotetext{
${ }^{4}$ Vale dizer que incorporando também a simetria por translações, chegamos à simetria de Poincaré, que corresponde ao conjunto fundamental de liberdades que um observador tem para escolher um referencial inercial. Como nos desenvolvimentos que buscamos descrever nesse trabalho nenhuma modificação é suposta no que concerne às translações, iremos sempre nos referir a simetria de Lorentz como a simetria fundamental do espaço-tempo na RR.
}

dida como uma generalização da idéia de rotações para o espaço-tempo: além das rotações usuais, envolvendo os eixos coordenados $x, y, z$, os "empurrões" podem ser vistos como uma espécie de rotação envolvendo o eixo dos tempos e os eixos coordenados 21,22, correspondendo fisicamente à relação entre dois referenciais que se movem com velocidade relativa constante.

A partir do reconhecimento da simetria de Lorentz como simetria fundamental do espaço-tempo, toda a estrutura da RR segue, e portanto esta simetria vale como um ponto de partida logicamente consistente para se desenvolver a RR. Livros introdutórios costumam construir a teoria de outra forma, mais próxima do seu desenvolvimento histórico, partindo dos problemas da teoria do éter eletromagnético e da resolução proposta por Einstein, baseado nos postulados da constância da velocidade da luz e do princípio da relatividade 23 .

A vantagem de tomar a simetria de Lorentz como ponto de partida é a centralidade do conceito de espaçotempo, e de suas simetrias. Percebendo essa centralidade, chegamos à relação direta que pode haver entre os princípios da RR e o problema da gravitação quântica: se a geometria do espaço-tempo pode ser modificada de forma radical na escala de Planck, seguirá a RR de forma inalterada nessa escala? Essa pergunta é o ponto chave deste artigo, e a principal motivação para o desenvolvimento do formalismo que iremos descrever.

A maioria dos pesquisadores que trabalham na área continuam adotando a RR como um ponto de partida não questionável, fato certamente motivado pelo sucesso extraordinário da RR como um dos fundamentos da física teórica moderna: tanto a RG quanto o MP tem como um de seus pilares fundamentais a RR. Desta forma, questionar a validade da $R R$ significa questionar a validade de boa parte da física moderna. Por outro lado, relembrando o argumento da seção2 como regra geral, imaginamos que toda teoria física tem seu domínio de validade. Ao explorarmos fenômenos físicos fora deste domínio, esta teoria pode se mostrar incorreta, e ser substituída por algo bastante diverso. No caso em questão, o domínio a que estamos nos referindo é essencialmente delimitado pela escala de Planck. Esperamos que uma nova física seja relevante nesta escala - porque aí é o domínio da gravitação quântica, que as leis que conhecemos até o momento tem se mostrado insuficientes para descrever. Essa nova física pode muito bem implicar em uma mudança na geometria e/ou no significado do espaço-tempo - e, portanto, da própria RR, cujos fundamentos estão profundamente ligados à sua descrição geométrica.

Algum tipo de modificação na simetria de Lorentz aparece em várias das abordagens propostas para a gravitação quântica até o momento. A RR é geralmente violada ou precisa ser modificada em várias propostas de geometria não-comutativa [24], assim como em abordagens em que o espaço-tempo é discretizado, sendo recuperada apenas em escalas de comprimento muito maiores que $\ell_{P}$. Já na teoria de cordas, a simetria de Lorentz pre- 
cisa ser parcialmente quebrada quando o espaço-tempo é reduzido das 26 ou 10 dimensões para as quatro dimensões usuais, embora geralmente se assuma que ela siga funcionando inalterada nessas quatro dimensões que correspondem ao que observamos em nosso cotidiano. A possibilidade de uma quebra espontânea da simetria de Lorentz, conceito que será melhor explicado na seção 5.2 . contudo, já foi apontada na literatura 25.

Afora o argumento descrito neste capítulo, que sugere a possibilidade de mudanças no espaço-tempo e em suas simetrias, temos também que lembrar um princípio mais elementar: testar os fundamentos de nossas teorias, principalmente quando avançamos para um domínio de fenômenos completamente novo, é parte crucial do fazer científico. Não sabemos de antemão se a simetria de Lorentz permanece válida na escala de Planck - o fato é que, apesar das muitas propostas teóricas, nenhuma informação experimental concreta existe sobre o que acontece nesta escala, e justamente por isso, testar a validade dos princípios mais básicos é um problema relevante.

Note que não estamos admitindo a hipótese de falhas da RR em fenômenos nas escalas "usuais", em que já testamos a física com grande precisão. Muitos já pretenderam encontrar paradoxos e inconsistências "óbvios" na RR - bem como na mecânica clássica, na termodinâmica, e na maioria das teorias consagradas da física - mas a verdade é que esses argumentos sempre se mostraram falhos, baseados muitas vezes numa incorreta compreensão da própria teoria que se pretende mostrar "falsa". Não estamos aqui dizendo que "Einstein está errado" e que portanto todos os livros de física teórica escritos a partir da primeira década do século XX precisam ser reescritos - estamos dizendo que a RR pode estar "errada" daquela forma muito peculiar descrita na seção2, Estamos dizendo que espera-se descobrir nova física na escala de Planck - e a simetria de Lorentz, fundamento essencial da RR, pode vir a ser uma peça essencial dela.

\section{Um modelo geral para violações de Lorentz: o Modelo Padrão Extendido}

Do ponto de vista teórico, a possibilidade da existência de violações da simetria de Lorentz é bastante interessante, dada a centralidade da RR nas teorias fundamentais para compreensão da física tanto no macro, quanto no microcosmo. Alterar de alguma forma os fundamentos da RR poderia ter consequências profundas, que deixariam os físicos teóricos ocupados por um bom tempo. Já do ponto de vista experimental, buscar por violações de Lorentz é um desafio enorme, visto a grande diferença de escalas envolvidas, conforme descrevemos na seção 3 . detectar efeitos que esperamos sejam tão pequenos, não é nada trivial. Justamente por isso, qualquer informação experimental sobre a física da escala de Planck - mesmo que seja uma confirmação de que a simetria de Lorentz continua valendo de forma exata - seria um dado nãotrivial.
De agora em diante, vamos detalhar um pouco mais aquele que tem se mostrado o mais útil formalismo para estudar esse problema, o chamado Modelo Padrão Extendido, ou SME (da expressão em inglês Standard Model Extension) [4,5]. Apresentaremos as idéias centrais que conduzem a esse formalismo, e as razões que o tornaram tão interessante para uma crescente comunidade de físicos teóricos e experimentais que se propõem a investigar a questão da sobrevivência da simetria de Lorentz na escala de Planck.

\subsection{Uma teoria efetiva para violações de Lorentz}

A primeira idéia fundamental é a de teoria efetiva. Para visualizar essa idéia, podemos imaginar um peixinho que vive dentro de um tanque de água que, por simplicidade, e seguindo o gosto dos físicos teóricos, consideramos infinito. Inicialmente, o peixinho olha para todas as direções, e enxerga o mesmo: apenas água, para todos os lados. Para ele, o universo parece ser feito de um meio contínuo, e é totalmente isotrópico: nada parece mudar se realizarmos uma rotação tendo como centro o peixinho. Para a maior parte das suas observações e experimentos, ele poderá usar a hidrodinâmica para explicar o que acontece ao seu redor. Se ele for versado em física e química, contudo, saberá que a realidade é mais complexa que isso: a verdade é que a água é feita de moléculas, que são entidades discretas, e que obedecem a uma física bastante diferente, a mecânica quântica. Acontece que essas moléculas são tão pequenas, e em tão grande número, que na prática o peixinho não as consegue identificar individualmente, e tudo se passa como se ele vivesse num meio contínuo, em que apenas propriedades médias (como densidade e pressão, por exemplo) existem. A descrição da água como um meio contínuo (com base na hidrodinâmica) funciona como uma teoria efetiva, válida para distâncias que são muito maiores do que o tamanho típico das moléculas o tamanho do peixinho, e de qualquer instrumento de laboratório que ele venha a usar. A física mais fundamental, que descreve as moléculas, pode ser bastante diferente da hidrodinâmica, contudo, essa física tem que se aproximar, quando observamos nas escalas de comprimento do peixinho, à hidrodinâmica que ele usa no seu dia-a-dia: qualquer efeito residual que possa existir do caráter discreto da água tem que ser muito pequeno, indetectável na maioria dos experimentos, exceto talvez naqueles de maior precisão.

Voltando ao nosso problema, raciocinamos como segue: por mais diferente que seja a física na escala de Planck, sabemos que se essa física é aplicada aos fenômenos que atualmente estudamos, ela precisa levar a resultados muito próximos aos das teorias que conhecemos, o MP e a RG. É muito razoável, portanto, supor que o MP e a RG sejam teorias efetivas, válidas nas escalas que atualmente conseguimos acessar, e que qualquer modificação residual dessa física mais fundamental seja presente através de 
correções bastante pequenas ao $\mathrm{MP}$ e à RG, que não esperamos detectar exceto em experimentos/observações de altíssima precisão.

O ponto de partida para a descrição de uma teoria da física fundamental é a prescrição de uma lagrangeana, que é uma função que depende dos vários campos existentes na teoria, a partir da qual basicamente toda a física é deduzida: as equações de movimento são obtidas através de um princípio variacional, pode-se descrever as simetrias e leis de conservação através do teorema de Noether, pode-se obter as regras de Feynman que servem de base a sua quantização, etc. A idéia de uma teoria efetiva para violações de Lorentz é que possamos tomar como base para nossos estudos uma lagrangena que seja da forma

$$
\mathcal{L}_{\text {eff }}=\mathcal{L}_{S M}+\mathcal{L}_{R G}+\mathcal{L}_{V L},
$$

onde $\mathcal{L}_{S M}$ é a lagrangeana correspondente ao MP (veja a figura 2), $\mathcal{L}_{R G}$ à $\mathrm{RG}$, e $\mathcal{L}_{V L}$ introduz novos termos, cuja origem deve ser a física desconhecida da escala de Planck. A física descrita pela soma $\mathcal{L}_{S M}+\mathcal{L}_{R G}$ é invariante por transformações de Lorentz, ao passo que vamos admitir que $\mathcal{L}_{V L}$ pode violar de alguma forma essa simetria. Essa violação deve ser bastante pequena, o que significa na prática que todo termo contido em $\mathcal{L}_{V L}$ deve conter algum coeficiente não determinado, e que vamos assumir ser muito pequeno - caso contrário, violações de Lorentz já teriam sido encontradas corriqueiramente nos experimentos que realizamos atualmente.

Do ponto de vista teórico, a questão é: quais termos podemos considerar em $\mathcal{L}_{V L}$, que por sua vez induzem a uma física que ainda faça sentido? Quais outras propriedades fundamentais podem ser perturbadas por essas

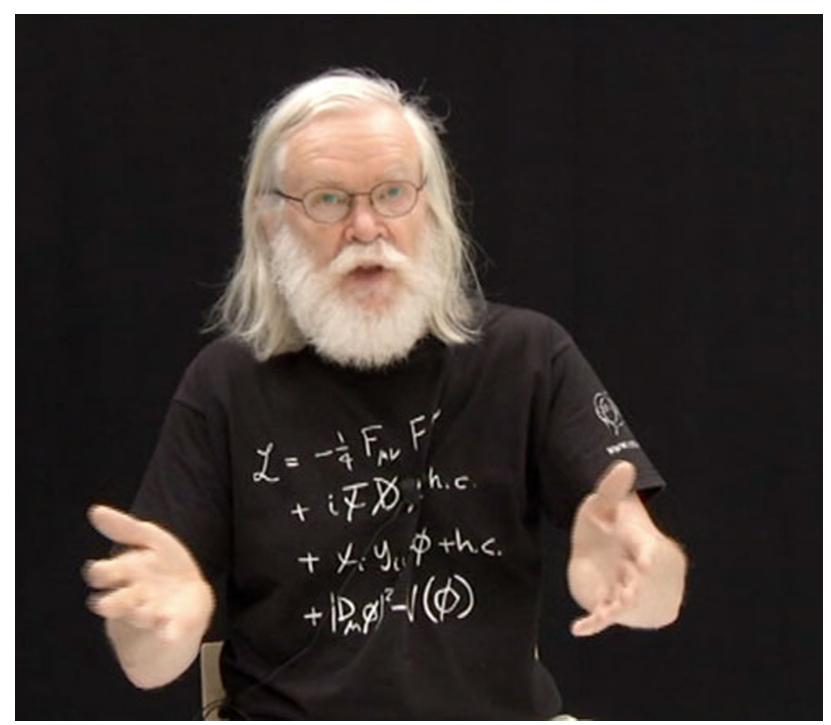

Figura 2: Uma lagrangiana é uma função que serve de ponto de partida para a descrição de um teoria fundamental da física como o MP. Na figura, o físico teórico John Ellis explica o mecanismo de Higgs usando uma camiseta onde aparece escrita, de forma sintética, a lagrangeana do MP. Vídeo disponível em: https://www.youtube.com/watch?v=QG8g5JW64BA. correções? Já do ponto de vista experimental, podemos tomar $\mathcal{L}_{\text {eff }}$ como ponto de partida, e reproduzir os cálculos relevantes para chegar às previsões do MP e/ou da RG para um dado experimento ou observação, só que os resultados serão agora corrigidos pelos pequenos termos presentes em $\mathcal{L}_{V L}$. Descobrir uma violação de Lorentz significaria realizar uma medida experimental que convincentemente provasse que alguma dessas correções de fato existe na natureza, o que significa que algum dos coeficientes presentes em $\mathcal{L}_{V L}$ é pequeno, mas não nulo. Por outro lado, enquanto todos os experimentos se mostrem consistentes com a simetria de Lorentz, o tamanho das incertezas das nossas medidas dá um dimensão do tamanho máximo que esses coeficientes desconhecidos podem ter - ou seja, a violação de Lorentz tem que ser menor que um determinado valor, senão nos já a teríamos percebido. Se, após décadas de experimentos, os resultados indicam que todos esses coeficientes são suficientemente pequenos, esse resultado poderia ser interpretado como uma evidência experimental sólida de que a simetria de Lorentz continua válida, mesmo na escala de Planck.

A noção de teoria efetiva é importante para o desenvolvimento do SME por uma série de razões. Sendo baseada no MP e na RG, os quais ela estende incorporando novos efeitos da violação de Lorentz, a teoria efetiva pode ser aplicada em praticamente qualquer experimento ou observação, desde que estes tenham precisão suficiente para detectar o tipo de efeito minúsculo que esperamos, já que o MP e a RG englobam todo o nosso conhecimento atual acerca de física fundamental. Incorporar as violações de Lorentz como pequenas correções sobre a física que conhecemos é consistente com nossa expectativa de que a RR certamente funciona muito bem nas escalas que atualmente conseguimos testar, e por isso qualquer desvio dela precisa ser incrivelmente pequeno. Conseguimos também, utilizando a idéia de teoria efetiva, preservar várias das demais simetrias fundamentais da natureza - de fato, este requerimento serve como um dos principais guias para se construir os possíveis termos presentes em $\mathcal{L}_{V L}$. Essa abordagem permite, em resumo, alcançar uma grande generalidade, mas ainda mantendo-se sob controle as consequências da violação de Lorentz, estabelecendo um formalismo que engloba possíveis efeitos de física nova na escala de Planck, sendo ainda aplicável nas escalas que efetivamente conseguimos testar nos laboratórios.

\subsection{Quebra espontânea de simetria}

Voltamos à nossa imagem do peixinho no tanque infinito de água. Quando as temperaturas estão acima do ponto de congelamento da água, essas moléculas estão posicionadas e orientadas de forma completamente aleatória, sem nenhuma direção preferencial, por isso, na prática, todas as direções são equivalentes. Como consequência, na teoria efetiva (que descreve a água como um líquido), o peixinho vê um meio isotrópico ao seu redor. Contudo, 
vamos assumir que (após o peixinho equipar-se de uma roupa protetora adequada) a temperatura seja baixada até ultrapassar o ponto de congelamento da água. $\mathrm{O}$ peixinho irá se ver dentro de um meio bastante diferente, o gelo. Acontece que, no gelo, as moléculas se organizam de uma forma regular (ou cristalina), e a rigor já não há isotropia, pelo menos não na descrição microscópica: rotações por ângulos arbitrários não deixam a rede cristalina invariante, apenas certas rotações por ângulos específicos, que dependem da forma exata como as moléculas se organizam. Temos assim o surgimento de direções preferenciais, e podemos imaginar que efeitos dessa redução de simetria podem surgir mesmo na física "macroscópica" (pense nos cristais de gelo da figura 3 que formam a neve, com suas estruturas tão belas, regulares, mas não esfericamente simétricas!).

Essa redução de simetria - no caso, devido à transição de fase da água líquida para o gelo - é um exemplo de quebra espontânea de simetria: as leis fundamentais que regem o comportamento das moléculas de água possuem simetria esférica, contudo, a interação entre diferentes moléculas é tal que, a baixas temperaturas, estas se organizam de uma forma particular (uma rede cristalina) que possui simetria menor do que a das leis fundamentais. No momento da transição de fase, as moléculas de água precisam escolher as direções em que vão formar sua rede cristalina e, devido a essa escolha, a simetria rotacional é parcialmente quebrada.

O mecanismo de quebra espontânea de simetria também acontece no Modelo Padrão, embora aí ele seja mais abstrato, envolvendo a quebra da chamada simetria de calibre. O ponto chave do mecanismo, nesse caso, é a existência do campo de Higgs, que induz a quebra de simetria na teoria 5 O bóson de Higgs é uma partícula escalar, o que significa que suas propriedades não depen-

\footnotetext{
${ }^{5}$ Outra consequência do campo de Higgs é a existência de uma partícula associada, o chamado bóson de Higgs, cuja descoberta pelo LHC décadas depois da sua formulação teórica foi bastante celebrada em 2012, como a última "peça" faltante para confirmar a validade do MP na descrição das interações nucleares e eletromagnéticas.
}

dem da orientação. Essa escolha, além de ser conveniente pela simplicidade, também tem outra razão: no MP, não queremos violações da simetria de Lorentz, justamente por isso é essencial que o campo responsável pela indução da quebra de simetria seja um escalar. O que mudaria, contudo, se imaginássemos que um campo que não fosse um escalar assumisse um papel similar ao do campo de Higgs? Um campo vetorial, por exemplo? Essa é justamente a idéia por trás da quebra espontânea da simetria de Lorentz: assumimos a existência de campos tensoriais (pense em vetores, como um caso particular mais intuitivo) que induzem uma quebra de simetria, similar à do campo de Higgs. Nesse caso, esses campos tensoriais assumirão valores constantes, que definem direções preferenciais no espaço vazio e, portanto, quebram a simetria de Lorentz.

O interessante dessa proposta é que, neste caso, a violação de Lorentz pode ser considerada menos "radical", no sentido de que a teoria fundamental da escala de Planck até pode ser invariante por Lorentz, mas o estado de menor energia da teoria, que representa o "espaço vazio", tem simetria menor que o da teoria fundamental: ele apresenta direções preferenciais representadas por coeficientes que são tensoriais, ou seja, vetores ou suas generalizações, e que são justamente os coeficientes de violação de Lorentz presentes na lagrangeana $\mathcal{L}_{V L}$ que apresentamos na seção 5.1 .

Há outra consequência, mais sutil, da suposição de que a violação de Lorentz seja espontânea, e é justamente o fato de que esses coeficientes são vetores ou tensores em geral. A simetria do espaço-tempo limita os tipos de objetos que podem aparecer numa teoria invariante por esta simetria: no caso da RR, pode-se mostrar que todas as teorias relativísticas devem envolver campos que sejam escalares, vetores ou, mais geralmente, tensores. Se abrimos mão completamente da simetria de Lorentz, não há razão porque imaginar que apenas objetos desses tipos possam aparecer na teoria, o que complicaria muito a escrita dos possíveis termos em $\mathcal{L}_{V L}$ - teríamos um número tão grande de possibilidades que estudos siste-
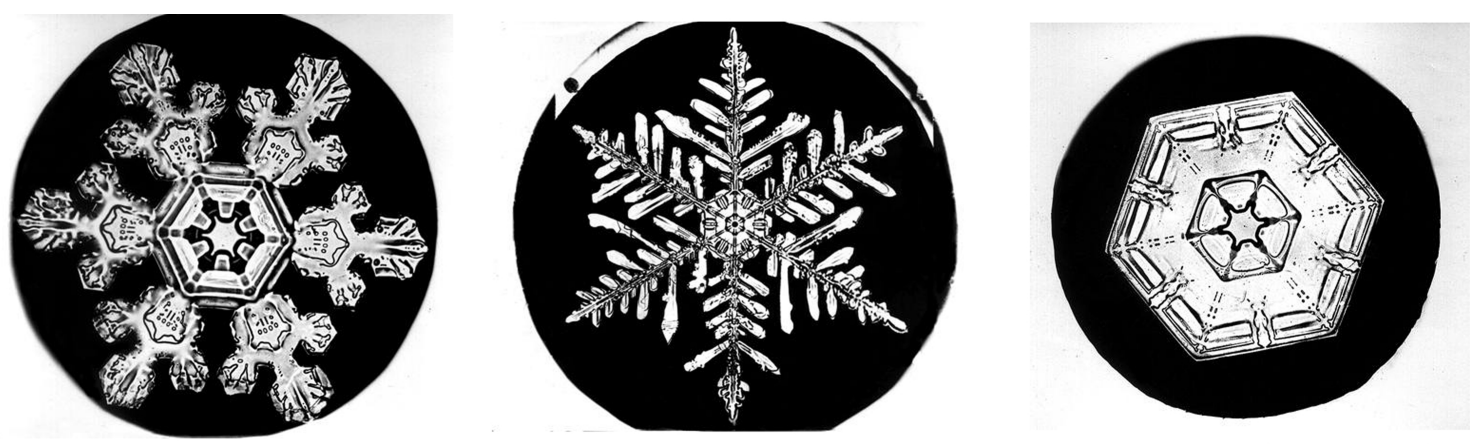

Fotos: Wilson Bentley

Figura 3: Fotografias de cristais de neve, obtidas por Wilson Bentley ainda no século XIX. Os cristais não possuem simetria esférica (ou seja, não são invariantes frente a rotações por ângulos arbitrários) devido à estrutura cristalina das moléculas de água: contudo, a quebra de simetria rotacional não é total, já que os cristais ainda herdam uma simetria parcial, por rotações de $\pi / 3$ radianos. 
máticos seriam bastante difíceis. Se a quebra de Lorentz é espontânea, contudo, os novos objetos que aparecem nessa teoria ainda podem ser classificados como tensores, o que restringe as possibilidades do que pode aparecer em $\mathcal{L}_{V L}$

\subsection{Transformações de Lorentz de observador e de partícula}

A suposição de quebra espontânea da simetria de Lorentz também leva naturalmente à distinção entre transformações de Lorentz de observador e de partícula [26], que é crucial no desenvolvimento do SME. Por simplicidade, vamos considerar no que segue rotações no plano $x y$, mas essa mesma idéia vale para todas as transformações de Lorentz, ou seja, o conjunto de três rotações espaciais e três "empurrões", caracterizando portanto a totalidade da simetria do espaço-tempo na RR.

Consideremos um dado ponto $p$, que será descrito por coordenadas cartesianas $(x, y)$ em um dado referencial arbitrário $S$. Ao considerar uma rotação no plano $x y$, temos basicamente duas formas de descrever essa transformação: no chamado ponto de vista passivo, imaginamos que o ponto $p$ permanece parado, mas o sistema de coordenadas $S$ é girado, tornando-se $S^{\prime}$; já no ponto de vista ativo, imaginamos que o referencial permanece o mesmo, mas o sistema sob consideração (no caso, o ponto $p$ ) é girado pelo mesmo ângulo, em sentido oposto. Em ambos os casos, as coordenadas para o ponto $p$ mudam para $\left(x^{\prime}, y^{\prime}\right)$, cuja expressão explícita pode ser encontrada com um pouco de trigonometria. Geometricamente, as duas transformações, representadas esquematicamente na figura 4 são equivalentes do ponto de vista de um observador que observa o ponto $p$ usando os referenciais indicados.

Quando pensamos no que significa, fisicamente, dizer que uma teoria é invariante por rotações no plano $x y$, contudo, as duas transformações representam aspectos ligeiramente diferentes. Na transformação passiva, temos
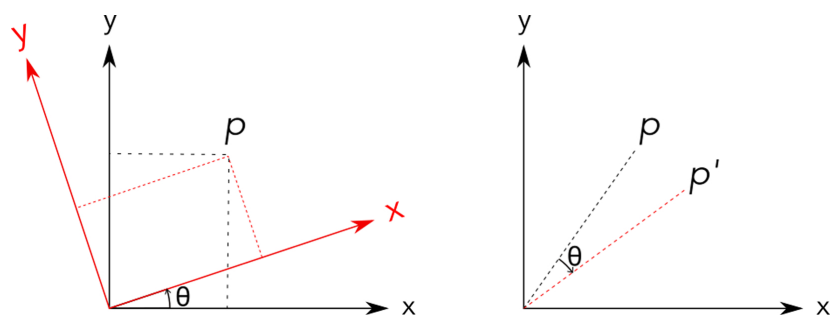

Figura 4: Visão passiva (esquerda) e ativa (direita) de uma mesma transformação, no caso uma rotação por ângulo $\theta$. $\mathrm{Na}$ visão passiva, o ponto $p$ fica parado, enquanto o referencial gira no sentido anti-horário. Na visão ativa, o ponto $p$ é girado no sentido horário pelo mesmo ângulo. A equivalência das duas descrições pode ser visualizada se pensamos sob o ponto de vista de um observador que esteja fixo sobre o referencial que é girado: o que ele enxerga é o ponto girando pelo mesmo ângulo, mas no sentido oposto. uma mudança de escolha de referencial, e como acreditamos que qualquer escolha de referencial é, como o próprio termo indica, uma escolha que não carrega significado físico, é difícil admitir uma teoria física que não seja invariante por transformações passivas. Já para transformações ativas, o referencial (arbitrário) $S$ permanece imutável, e o que fazemos é girar o sistema físico considerado. Neste caso, o que estamos investigando é se o espaço possui direções preferenciais, o que detectaríamos como experimentos apresentando resultados diferentes conforme giramos o sistema físico que, de princípio, inclui todas as partículas e fontes de campos que são relevantes. A invariância da física por transformações ativas, portanto, representa a idéia da isotropia do universo e das leis da física.

No contexto das teorias com violação de Lorentz que estamos discutindo, vamos considerar essas duas diferentes visões de uma transformação como independentes, levando em conta a interpretação física delineada no último parágrafo, e para evitar confusões, elas receberão nomes distintos: as transformações que correspondem a uma rotação do referencial serão chamadas de transformações de observador, enquanto que aquelas correspondendo a uma rotação do sistema serão chamadas de transformações de partícula. Queremos incorporar nas teorias conhecidas pequenas violações da simetria rotacional. Não queremos contudo perder a idéia de invariância da física frente à escolha de um referencial, de forma que vamos manter a invariância por transformações de observador. Restará, portanto, admitir a possibilidade de pequenas violações da invariância frente às transformações de partícula, ou seja, violações na isotropia das leis da física. Isso significa que, diante de rotações do laboratório, girando de forma idêntica todas as partículas e campos relevantes ao experimento considerado, encontraremos resultados diferentes dependendo do ângulo de rotação, devido a uma anisotropia fundamental no universo.

É interessante perceber que o cenário aqui descrito aparece naturalmente se supomos que a quebra da simetria de Lorentz ocorre de forma espontânea, conforme explicado na subseção anterior. De qualquer forma, se tentamos formular matematicamente a teoria efetiva com violações de Lorentz, essas idéias reduzem drasticamente os termos que podemos escrever em $\mathcal{L}_{V L}$ : para preservar a invariância por transformações de observador, possíveis contribuições para $\mathcal{L}_{V L}$ devem ser escritas basicamente obedecendo as mesmas regras que garantem a escrita de lagrangeanas invariantes pela RR ${ }^{6}$ Por outro lado, para violar a invariância por transformações de partículas, temos que assumir que $\mathcal{L}_{V L}$ contêm coeficientes vetoriais (ou tensoriais) que não representam campos ou partículas, e que não se transformam da forma esperada frente a uma transformação de partícula: o mais simples é assumir que esses coeficientes ficam invariantes enquanto os campos físicos giram. No caso mais simples

${ }^{6} \mathrm{Ou}$ seja, contribuições para $\mathcal{L}_{V L}$ devem ser escritas em termos de tensores com todos os índices contraídos de forma relativisticamente covariante. 
de um vetor, que denotamos por $k^{\mu / 7}$ isso significa que um termo possível para $\mathcal{L}_{V L}$ seria por exemplo

$$
\mathcal{L}_{V L} \supset k^{\mu} \mathcal{O}_{\mu}(\phi, \psi, A, \cdots),
$$

onde $\mathcal{O}_{\mu}(\phi, \psi, A, \cdots)$ significa uma expressão envolvendo campos físicos, que podem ser escalares, espinoriais, vetoriais, etc., que tenha um índice covariante $\mu$ sobrando para ser contraído com o coeficiente $k^{\mu}$. Frente a uma transformação de observador, tanto $k^{\mu}$ quanto $\mathcal{O}_{\mu}$ se transformam da forma usual, ou seja, de maneira que a transformação de um dos fatores cancele exatamente a transformação do outro, garantindo que $\mathcal{L}_{V L}$ seja invariante. Contudo, numa transformação de partícula, apenas os campos giram, de forma que $\mathcal{O}_{\mu}$ se transforma, ao passo que $k^{\mu}$ permanece invariante, e por consequência $\mathcal{L}_{V L}$ não é invariante frente a essas transformações. Podemos interpretar $k^{\mu}$ como definindo uma direção preferencial no "espaço vazio", e a presença de um termo como o descrito na última equação representa um acoplamento dos campos físicos à essa direção, de forma que o resultado de experimentos vai depender da orientação do laboratório com relação a essa direção preferencial - essa situação está representada esquematicamente na figura 5 .

\section{O eletromagnetismo com violações de Lorentz}

Com todas as idéias básicas apresentadas, podemos finalmente estudar o seguinte problema: qual a teoria efetiva mais geral que podemos escrever, incorporando violação de Lorentz, a partir de uma certa parte do MP? Vamos responder à essa pergunta considerando a parte mais bem conhecida do MP, aquela que descreve o campo eletromagnético.

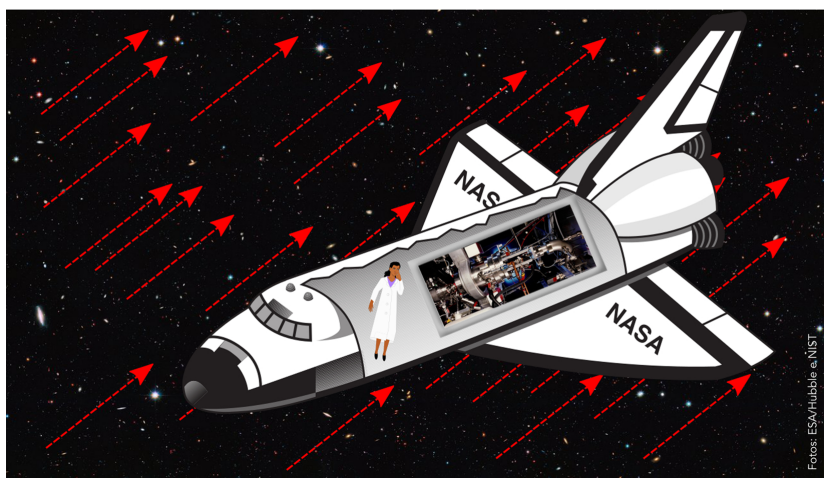

Figura 5: Uma cientista realiza experimentos com um equipamento altamente sensível no espaço, longe de qualquer campo gravitacional ou eletromagnético externo que pudesse influenciar seus resultados. Mesmo assim, conforme gira seu laboratório, ela percebe diferenças em suas medidas físicas, devido à presença da violação de Lorentz, que define direções preferenciais (representadas pelas setas vermelhas) mesmo na ausência de qualquer campo externo.

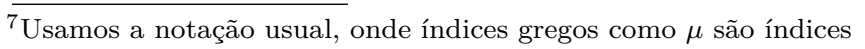
do espaço-tempo, assumindo os valores $\mu=0,1,2,3$.
Vamos primeiro rever como escrever através de uma lagrangena $\mathcal{L}_{E D}$ a teoria da eletrodinâmica (ED) na ausência de cargas e fontes. Queremos, enfim, uma função $\mathcal{L}_{E D}$ que envolva os campos elétricos e magnéticos $\vec{E} \mathrm{e}$ $\vec{B}$, tais que as equações de movimento sejam as equações de Maxwell no vácuo,

$$
\nabla \cdot \vec{B}=\nabla \cdot \vec{E}=0 ; \nabla \times \vec{E}+\frac{\partial \vec{B}}{\partial t}=\nabla \times \vec{B}-\frac{\partial \vec{E}}{\partial t}=0 .
$$

Note que por simplicidade escrevemos essas equações em unidades naturais (o leitor pode aprender mais sobre essa escolha em [16]). Como essas equações são lineares nos campos, a correspondente lagrangeana precisa ser quadrática nesses mesmos campos. Além disso, como a ED respeita a simetria de Lorentz, precisamos impor que $\mathcal{L}_{E D}$ seja um escalar. Por fim, a teoria possui outra simetria que precisa ser levada em conta, a chamada simetria de calibre. Essa simetria aparece quando escrevemos os campos $\vec{E}$ e $\vec{B}$ em termos de potenciais escalar $\phi$ e vetor $\vec{A}$, e percebemos que diferentes potenciais representam os mesmos campos físicos $\vec{E}$ e $\vec{B}$.

Todos os requerimentos acima são resolvidos da seguinte maneira: para respeitar a invariância relativística, os potenciais $\phi$ e $\vec{A}$ são aglomerados em um único vetor $A^{\mu}=(\phi, \vec{A})$. A transformação de calibre da ED se escreve, para $A^{\mu}$, da forma

$$
A^{\mu} \rightarrow A^{\mu}+\frac{\partial \Lambda}{\partial x_{\mu}},
$$

onde $\Lambda$ é uma função arbitrária. Começando com potenciais quaisquer $A^{\mu}$, podemos aplicar transformações desse tipo arbitrariamente, e os campos físicos não podem mudar. Para realizar isso, percebemos que uma derivada antissimetrizada do potencial, ou seja,

$$
F^{\mu \nu}=\frac{\partial A^{\nu}}{\partial x_{\mu}}-\frac{\partial A^{\mu}}{\partial x_{\nu}},
$$

é invariante frente à transformação de calibre. Portanto, se escrevemos $\mathcal{L}_{E D}$ como um escalar (ou seja, com todos os índices contraídos de forma covariante), quadrático em $F^{\mu \nu}$, garantimos todas as condições citadas. Introduzindo um coeficiente numérico adequado, somos levados a uma única estrutura possível

$$
\mathcal{L}_{E D}=-\frac{1}{4} F^{\mu \nu} F_{\mu \nu}
$$

e pode-se mostrar que dessa lagrangeana se derivam equações de movimento que reproduzem as equações de Maxwell, com as identificações

$$
E^{i}=F^{i 0}, B^{1}=-F^{23}, B^{2}=-F^{31}, B^{3}=-F^{12},
$$

onde $i=1,2,3$. A lagrangeana escrita em termos dos familiares campos $\vec{E}$ e $\vec{B}$ torna-se

$$
\mathcal{L}_{E D}=\frac{1}{2}\left(\vec{E}^{2}-\vec{B}^{2}\right) .
$$


Este raciocínio nos leva à teoria de Maxwell, partindo dos princípios gerais de simetria de Lorentz e de calibre, e da linearidade das equações de movimento. Este não é o caminho padrão pelo qual a teoria é apresentada em cursos elementares: geralmente, a discussão começa com os efeitos experimentais que levam à idéia dos campos elétricos e magnéticos, sua relação com as fontes (que estamos ignorando totalmente aqui) e, por fim, a conexão entre os campos $\vec{E}$ e $\vec{B}$ que é descoberta através da lei de Faraday e da modificação de Maxwell à lei de Ampère, resultando finalmente no conjunto completo das equações de Maxwell. Este caminho padrão tem a vantagem de ter uma relação bastante direta com os experimentos que dão suporte à definição das diferentes quantidades e suas relações, levando até a teoria completa do eletromagnetismo. Contudo, essa formulação não deixa evidente o fato das equações de Maxwell serem compatíveis com a RR, por exemplo. Além disso, a formulação do eletromagnetismo da forma como delineamos aqui pode ser generalizada, e suas generalizações encontram aplicações na descrição de outras interações da natureza.

A expressão para $\mathcal{L}_{E D}$ aqui obtida representa a forma mais compacta para escrever a teoria do eletromagnetismo, bem como aquela que deixa mais explícita as simetrias respeitadas pela teoria. O leitor pode até ver esse argumento como uma "dedução" do eletromagnetismo a partir das suas simetrias fundamentais, e apreciar sua simplicidade e mesmo "beleza" - desde que tenha em mente que essa "dedução" só faz sentido após a verificação experimental das consequências da teoria, que é a maneira definitiva de decidir o que é uma teoria física válida em algum domínio de fenômenos.

Voltando agora ao problema de incorporar a violação de Lorentz, seguindo os preceitos delineados nessa seção, queremos escrever

$$
\mathcal{L}_{S M E}^{E D}=\mathcal{L}_{E D}+\mathcal{L}_{V L}^{E D}
$$

onde $\mathcal{L}_{V L}^{E D}$ contem todos os termos que preservam ainda a simetria de calibre, mas incluem tensores constantes, que são os coeficientes que induzem a violação de Lorentz na teoria. Queremos também, numa primeira aproximação, considerar apenas termos quadráticos nos campos, o que preservará o caráter linear das equações de movimento. Essas restrições são bastante fortes, permitindo apenas duas estruturas possíveis: uma quadrática nos campos $F^{\mu \nu}$,

$$
\left(k_{F}\right)^{\mu \nu \rho \sigma} F_{\mu \nu} F_{\rho \sigma}
$$

e a outra é um termo proporcional à $\epsilon_{\mu \nu \rho \sigma} A^{\nu} F^{\rho \sigma}$, envolvendo o símbolo totalmente antissimétrico $\epsilon_{\mu \nu \rho \sigma}$, que pode-se mostrar é invariante de calibre a menos de um termo de superfície, ou seja,

$$
\left(k_{A F}\right)^{\mu} \epsilon_{\mu \nu \rho \sigma} A^{\nu} F^{\rho \sigma} .
$$

Falta agora escolher normalizações convenientes para esses dois termos, e chegamos assim à proposta introduzida em 5. como extensão mais geral da eletrodinâmica incluindo violações de Lorentz,

$$
\begin{aligned}
\mathcal{L}_{S M E}^{E D} & =-\frac{1}{4} F^{\mu \nu} F_{\mu \nu}+\frac{1}{4}\left(k_{F}\right)^{\mu \nu \rho \sigma} F_{\mu \nu} F_{\rho \sigma} \\
& +\frac{1}{2}\left(k_{A F}\right)^{\mu} \epsilon_{\mu \nu \rho \sigma} A^{\nu} F^{\rho \sigma} .
\end{aligned}
$$

O vetor constante $\left(k_{A F}\right)^{\mu}$ claramente define uma direção preferencial no espaço-tempo, violando a simetria de Lorentz. O mesmo vale para o tensor $\left(k_{F}\right)^{\mu \nu \rho \sigma}$, que de princípio teria $4^{4}$ componentes independentes, mas após o estudo das simetrias impostas pela consistência da teoria, mostra-se que possui 19 componentes independentes. Existem várias maneiras de parametrizar essas componentes, uma delas em termos de matrizes $3 \times 3$ denominadas $\kappa_{D E}, \kappa_{H B}$ e $\kappa_{D B}$ (o leitor pode consultar o artigo 27 para as definições) tais que a teoria do eletromagnetismo incluindo as correções envolvendo $k_{F}$ escreve-se

$$
\begin{aligned}
\left.\mathcal{L}_{S M E}^{E D}\right|_{k_{A F}=0} & =\frac{1}{2}\left(\vec{E}^{2}-\vec{B}^{2}\right)+\frac{1}{2} \vec{E} \cdot \kappa_{D E} \cdot \vec{E} \\
& -\frac{1}{2} \vec{B} \cdot \kappa_{H B} \cdot \vec{B}+\vec{E} \cdot \kappa_{D B} \cdot \vec{B}
\end{aligned}
$$

O primeiro termo corresponde à ED usual, os termos adicionais incorporam a violação de Lorentz. As equações de Maxwell obtidas desta teoria podem se escrever da seguinte forma,

$$
\nabla \cdot \vec{D}=\nabla \cdot \vec{B}=0
$$

$$
\nabla \times \vec{E}+\frac{\partial \vec{B}}{\partial t}=\nabla \times \vec{H}-\frac{\partial \vec{D}}{\partial t}=0
$$

onde

e

$$
\begin{aligned}
& \vec{D}=\left(1+\kappa_{D E}\right) \cdot \vec{E}+\kappa_{D B} \cdot \vec{B} \\
& \vec{H}=\left(1+\kappa_{H B}\right) \cdot \vec{B}-\kappa_{D B} \cdot \vec{E} .
\end{aligned}
$$

O leitor deverá reconhecer, nessas últimas equações, algo similar ao que chamamos de eletromagnetismo na presença de um meio dielétrico: a presença de polarização e magnetização no meio leva à conveniência de se reescrever as equações de Maxwell em termos dos campos $\vec{D}$ e $\vec{H}$, e a uma série de efeitos bem conhecidos como a birrefringência, quando a velocidade de propagação da luz é diferente para diferentes polarizações da onda luminosa (discutiremos em breve um exemplo específico desse fenômeno). No caso com violação de Lorentz, esses efeitos poderiam estar presentes mesmo no vácuo, o que seria uma consequência dramática da existência da anisotropia do espaço-tempo.

O modelo aqui descrito é o mais geral capaz de descrever pequenos efeitos de violação de Lorentz em fenômenos eletromagnéticos. Pensando em termos de partículas numa hipotética teoria quântica incorporando gravitação, dizemos que, seja qual for a nova física na escala de Planck e seu efeito sobre fótons, ao se considerar fenômenos em 
mais baixas energias - comparadas à $E_{P}$ - esperamos uma física que possa ser descrita por uma lagrangeana da forma $\mathcal{L}_{S M E}^{E D}$ : esta é justamente a idéia de uma teoria efetiva. Propostas particulares de nova física, que levam de alguma forma a violações de Lorentz, devem em algum limite se reduzir à lagrangeana $\mathcal{L}_{S M E}^{E D}$, com alguma identificação adequada dos coeficientes $k_{A F}$ e/ou $k_{F}$ : um exemplo são as chamadas teorias não-comutativas, que foram muito estudadas na primeira década deste século (o leitor encontra em [28] uma introdução à teorias nãocomutativas, e em 24] é discutida sua relação com o formalismo do SME). Já do ponto de vista experimental, a lagrangeana $\mathcal{L}_{S M E}^{E D}$ pode ser entendida como uma parametrização geral dos possíveis efeitos de violação de Lorentz no eletromagnetismo, e experimentos podem ser desenvolvidos buscando-se medir cada um dos coeficientes presentes em $\mathcal{L}_{S M E}^{E D}$, conforme veremos nos exemplos a seguir.

\subsection{Um exemplo: eletrodinâmica na presença de um vetor constante $\vec{\beta}$}

Um exemplo simples e útil para fins didáticos, descrito em [5], consiste na escolha particular de $k_{F}$ tal que $\left(k_{F}\right)_{0 i 0 j}=-\frac{1}{2} \beta_{i} \beta_{j}$, para $i=1,2,3$, com todas as demais componentes de $k_{F}$ sendo nulas, bem como as de $k_{A F}$. Neste caso, a violação de Lorentz é descrita por um único vetor $\vec{\beta}$ que define uma direção preferencial no espaço. Com esta escolha, a única das matrizes $\kappa$ diferente de zero é $\left(\kappa_{D E}\right)_{i j}=\beta_{i} \beta_{j}$, e como consequência, a lagrangeana efetiva se reduz a

$$
\left.\mathcal{L}_{S M E}^{(E D)}\right|_{\vec{\beta}}=\frac{1}{2}\left(\vec{E}^{2}-\vec{B}^{2}\right)+\frac{1}{2}(\vec{\beta} \cdot \vec{E})^{2} .
$$

As equações de Maxwell modificadas obtidas nesta teoria, na ausência de fontes, são

$$
\nabla \cdot \vec{E}=-\beta \cdot \vec{\nabla}(\beta \cdot \vec{E})
$$

e

$$
\nabla \times \vec{B}-\frac{\partial \vec{E}}{\partial t}=\vec{\beta} \frac{\partial}{\partial t}(\beta \cdot \vec{E}) .
$$

Essas equações governam a propagação de ondas luminosas no vácuo, e um estudo dessas ondas mostra a existência de duas possíveis polarizações, ou modos de propagação, um chamado de "ordinário" obedecendo à relação usual entre frequência $p^{0}=\omega$ e número de onda $\vec{p}=\vec{h}$, ou seja,

$$
\left(p^{0}\right)_{o}^{2}-\left(\vec{p}_{o}\right)^{2}=0
$$

\footnotetext{
${ }^{8} \mathrm{O}$ leitor deve relembrar que, usualmente, a relação entre o vetor momento associado à uma onda de frequência $\omega$ e número de onda $\vec{k}$ é $p^{\mu}=\left(p^{0}, \vec{p}\right)=\hbar\left(\frac{\omega}{c}, \vec{k}\right)$, contudo como estamos considerando unidades naturais em que $\hbar=c=1$, temos simplesmente $p^{\mu}=$ $(\omega, \vec{k})$.
}

enquanto outra polarização, chamada de "extraordinária", satisfaz

$$
\left(p^{0}\right)_{e}^{2}-\left(\vec{p}_{e}\right)^{2}=-\frac{\left(\vec{\beta} \times \vec{p}_{e}\right)^{2}}{1+\vec{\beta}^{2}} .
$$

Claramente, essa segunda polarização tem, de "extraordinário", uma dependência da sua velocidade de fase com a orientação entre seu vetor de onda $\vec{p}_{e}$ e o vetor $\vec{\beta}$. Note que esse efeito desaparece caso a onda se propague na mesma direção de $\vec{\beta}$, e é máximo quando a onda se propaga perpendicularmente a $\vec{\beta}$. Vale lembrar que a relação entre frequência e número de onda é fundamental no estudo de propagação de ondas, e recebe o nome de relação de dispersão. Dizemos, portanto, que uma das polarizações da luz tem uma relação de dispersão modificada pela violação de Lorentz. Esse fato tem consequências experimentais para a propagação de ondas eletromagnéticas, que poderiam ser estudadas experimentalmente [29].

A modificação na relação de dispersão aqui descrita implica na existência de raios de luz com velocidade diferente da constante $c=1$ (lembre-se que estamos usando unidades naturais). Um estudo completo dessas teorias implica, por exemplo, em investigar se problemas envolvendo causalidade podem surgir decorrentes deste fato. No presente exemplo, vamos estar interessados no caso em que a modificação devida à violação de Lorentz é máxima, ou seja, $\vec{p} \perp \vec{\beta}$. Neste caso, $\left(\vec{\beta} \times \vec{p}_{e}\right)^{2}=$ $(\vec{\beta})^{2}\left(\vec{p}_{e}\right)^{2}$ e com isso pode-se mostrar que a relação de dispersão extraordinária se reduz a

$$
p^{0}=\frac{\left|\vec{p}_{e}\right|}{\sqrt{1+(\vec{\beta})^{2}}}
$$

o que significa que a velocidade de propagação desse modo é $v=1 / \sqrt{1+(\vec{\beta})^{2}}$. Note que neste caso, $v<1$ para qualquer valor não-nulo do vetor $\vec{\beta}$, então em princípio não esperamos problemas de causalidade.

Imaginemos que a natureza apresentasse de fato violação de Lorentz, conforme descrita nesse exemplo. Neste caso, poderíamos fazer experimentos com lasers ou outras fontes luminosas, tomando sempre cuidado de especificar a direção exata em que se propagam os raios de luz, porque os resultados vão depender da orientação destes com relação a um certo vetor $\vec{\beta}$, que de princípio desconhecemos. Imaginemos agora uma medida com um raio de luz fixo na Terra, mas que é repetida várias vezes ao longo de vários dias. Neste caso, como a Terra gira ao redor do seu próprio eixo, carregando consigo o laboratório, a orientação das nossas fontes em relação ao vetor $\vec{\beta}$ vai variar periodicamente, com um período de aproximadamente 24h. Esse já seria um primeiro efeito de teorias com violação de Lorentz: medidas físicas devem oscilar 
com uma frequência aproximada de $24 \mathrm{~h}$, devido à rotação da Terra 9

Pensemos agora em outro tipo de medida, em que procuramos detectar os efeitos de birrefringência que foram citados anteriormente. Para entender como esse efeito irá aparecer nesse exemplo, vamos relembrar alguns conceitos sobre a polarização de ondas eletromagnéticas. Assumimos por simplicidade que $\vec{p}$ está na direção $x$, e consideramos dois vetores unitários, mutuamente perpendiculares, $\vec{\epsilon}_{1}$ e $\vec{\epsilon}_{2}$, no plano $y z$. Desta forma, uma onda eletromagnética com polarização plana seria descrita por um potencial da forma

$$
\vec{A}_{p}(\vec{x}, t)=\left(c_{1} \vec{\epsilon}_{1}+c_{2} \vec{\epsilon}_{2}\right) e^{i\left(\vec{p} \cdot \vec{x}-p^{0} t\right)},
$$

onde $c_{1}, c_{2} \in \mathbb{R}$. Por comodidade, considerando a geometria considerada para o problema, podemos reescrever a exponencial presente nessa expressão como $e^{i p^{0}(\alpha|\vec{p}| x-t)}$, onde

$$
\alpha=|\vec{p}| / p^{0} .
$$

Lembramos também que estamos usando a conveniente técnica de considerar exponenciais complexas ao invés de funções trigonométricas, o que significa que o resultado final deve ser sempre obtido tomando-se a parte real do potencial complexo $A^{10}$

Para visualizar fisicamente o que significa esse potencial, considere um ponto fixo do espaço, por exemplo $x=0$. Neste caso, temos

$$
\vec{A}_{p}(x=0, t)=f\left(c_{1} \vec{\epsilon}_{1}+c_{2} \vec{\epsilon}_{2}\right) e^{-i p^{0} t},
$$

onde $f$ é uma fase complexa e por simplicidade vamos assumir $f=1$. Tomando-se por fim a parte real, obtemos o potencial

$$
\boldsymbol{\operatorname { R e }} \vec{A}_{p}(x=0, t)=\left(c_{1} \vec{\epsilon}_{1}+c_{2} \vec{\epsilon}_{2}\right) \cos \left(p^{0} t\right) .
$$

A expressão $\left(c_{1} \vec{\epsilon}_{1}+c_{2} \vec{\epsilon}_{2}\right)$ representa um vetor $\vec{A}_{0}$ fixo no plano perpendicular à propagação da onda, e o que temos é um potencial que oscila sempre na linha definida por esse vetor. Se calculamos a partir do potencial os campos eletromagnéticos, iremos encontrar campos perpendiculares entre si e com relação à $\vec{p}$, cada um deles oscilando numa direção fixa. Isso é o que chamamos de uma onda eletromagnética com polarização plana.

Se assumimos agora que os coeficientes $c_{1}, c_{2}$ são complexos, teremos uma situação bastante diferente. Por simplicidade, assumimos que $c_{1} \in \mathbb{R}$ e que no lugar de $c_{2}$ temos $i c_{2}$, com $c_{2} \in \mathbb{R}$, ou seja,

$$
\overrightarrow{A_{e}}(\vec{x}, t)=\left(c_{1} \vec{\epsilon}_{1}+i c_{2} \vec{\epsilon}_{2}\right) e^{i\left(\vec{p} \cdot \vec{x}-p^{0} t\right)} .
$$

\footnotetext{
${ }^{9}$ Efeito similar, contudo, de intensidade muito menor, também deve vir da rotação da Terra ao redor do Sol.

${ }^{10}$ Note também que estamos discutindo ondas em termos do vetor potencial $\vec{A}$ e não dos campos físicos $\vec{E}$ e $\vec{B}$. Esta escolha é uma mera questão de conveniência, e nenhuma mudança nas conclusões a que chegaremos viria do estudo dos campos eletromagnéticos diretamente.
}

Neste caso, novamente olhando para o plano $x=0$ e assumindo $f=1$, temos

$$
\begin{aligned}
\vec{A}_{e}(x=0, t) & =\left(c_{1} \vec{\epsilon}_{1}+i c_{2} \vec{\epsilon}_{2}\right) e^{-i p^{0} t} \\
& =\left(c_{1} \vec{\epsilon}_{1} e^{-i p^{0} t}+c_{2} \vec{\epsilon}_{2} e^{-i\left(p^{0} t-\pi / 2\right)}\right),
\end{aligned}
$$

e, tomando a parte real,

$$
\operatorname{Re} \vec{A}_{e}(x=0, t)=c_{1} \vec{\epsilon}_{1} \cos \left(p^{0} t\right)-c_{2} \vec{\epsilon}_{2} \sin \left(p^{0} t\right) .
$$

Neste caso, o vetor potencial não permanece numa única direção, mas sim gira conforme o tempo passa, em torno da direção de propagação dada por $\vec{p}$. Caso $c_{1}=c_{2}$, o vetor $\vec{A}$ descreve um círculo ao girar, por isso dizemos que a luz está circularmente polarizada, enquanto que no caso geral $c_{1} \neq c_{2}$, ela está elipticamente polarizada.

Vamos agora considerar o caso com a violação de Lorentz. Mantemos a direção de propagação da luz sobre o eixo dos $x$, e vamos assumir que $\vec{\beta}$ está na direção $y$. Neste caso, observamos que as duas relações de dispersão que encontramos implicam a existência de dois modos de propagação da luz, um modo ordinário com $\alpha_{o}=|\vec{p}| / p^{0}=1$ e um modo extraordinário, com $\alpha_{e}=\sqrt{1+\beta^{2}}$. Vamos imaginar que temos um emissor de luz capaz de emitir uma superposição desses dois modos de onda, da forma

$$
\vec{A}(\vec{x}, t)=c_{o} \vec{\epsilon}_{o} e^{i p^{0}(|\vec{p}| x-t)}+c_{e} \vec{\epsilon}_{e} e^{i p^{0}\left(\alpha_{e}|\vec{p}| x-t\right)},
$$

$\operatorname{com} c_{o}, c_{e} \in \mathbb{R}$. Em $x=0$, temos claramente uma onda polarizada de forma planar, correspondendo a uma combinação específica destes dois modos de propagação de luz. Considere agora o plano localizado em $x=\Delta$, com

$$
\Delta=\frac{\pi}{2\left(\sqrt{1+\beta^{2}}-1\right) p^{0}},
$$

pode-se mostrar que

$$
\vec{A}(x=\Delta, t)=f\left(c_{o} \vec{\epsilon}_{o}+i c_{e} \vec{\epsilon}_{e}\right) e^{-i p^{0} t},
$$

onde novamente $f$ é uma fase. Essa última expressão representa uma onda com polarização elíptica. Neste caso, a onda resultante oscila entre diferentes combinações do modo ordinário e extraordinário, um comportamento marcadamente distinto daquele que encontramos em $x=0$. Este seria um efeito da violação de Lorentz que esperaríamos detectar com experimentos adequados.

Muitos experimentos diferentes já foram feitos para tentar detectar a presença de coeficientes $k_{F}$ ou $k_{A F}$ na eletrodinâmica, e dados de experimentos já realizados puderam ser reinterpretados para obter informações sobre $k_{F}$ e $k_{A F}$. Como já adiantamos na introdução, nenhuma evidência convincente de violação de Lorentz - ou seja, um coeficiente não nulo - foi encontrada até hoje, e da margem de erro desses resultados nulos podemos inferir limites experimentais, ou seja, estabelecer valores máximos que coeficientes de $k_{F}$ e $k_{A F}$ poderiam assumir 
para serem consistentes com os dados experimentais. Esses limites mais recentes são encontrados em [30], uma compilação dos resultados acerca de coeficientes de violação de Lorentz que tem sido atualizada periodicamente. Considerando o modelo simplificado considerado nesta subseção, a forma particular de $k_{F}$ que assumimos implica em coeficientes não nulos para a matriz denominada $\tilde{\kappa}_{e-}$ e para a constante $\tilde{\kappa}_{t r}$ (veja 31] para as definições), e os limites experimentais mais recentes para esses coeficientes se encontram nas tabelas intituladas "Photon sector, $d=4$ " da referência [30]. O leitor pode apreciar a enorme variedade de experimentos diferentes que foram utilizados para estabelecer esses limites, que vão desde experimentos ópticos e atômicos até observações astrofísicas 11

\subsection{Um segundo exemplo: O modelo de Carroll-Field-Jackiw}

Outro exemplo, desenvolvido antes mesmo do formalismo completo do SME, foi descrito em [32], consistindo na escolha $k_{F}=0$. A teoria resultante, que chamaremos de modelo CFJ, é descrita pela lagrangeana

$$
\mathcal{L}_{C F J}=-\frac{1}{4} F^{\mu \nu} F_{\mu \nu}+\frac{1}{2}\left(k_{A F}\right)^{\mu} \epsilon_{\mu \nu \rho \sigma} A^{\nu} F^{\rho \sigma} .
$$

Uma motivação adicional para estudar esse modelo é o enorme grau de interesse gerado pela chamada teoria de Chern-Simons (CS), que é descrita por uma lagrangeana muito similar,

$$
\mathcal{L}_{C S}=-\frac{1}{4} F^{\mu \nu} F_{\mu \nu}+\frac{k}{2} \epsilon_{\nu \rho \sigma} A^{\nu} F^{\rho \sigma}
$$

só que essa teoria vive num espaço-tempo de três dimensões, ou seja, um tempo e dois espaços. A teoria de CS possui muitas aplicações em física, desde a descrição do efeito Hall e supercondutividade em materiais, além de propriedades teóricas como o fato do fóton possuir uma massa invariante de calibre 33. A generalização do modelo de CS para quatro dimensões espaço-temporais só é possível com a inclusão de um vetor constante, o que implica em uma violação da simetria de Lorentz. Ao invés de considerar isso como um defeito, que a tornaria uma teoria desinteressante, os autores do artigo 32 decidiram testar se essa violação de Lorentz poderia ter algum efeito observável, que pudesse ser comparado com experimentos.

Duas das equações de Maxwell nesta teoria são modificadas,

$$
\nabla \cdot \vec{E}=-\vec{k}_{A F} \cdot \vec{B}
$$

$\mathrm{e}$

$$
\nabla \times \vec{B}-\frac{\partial \vec{E}}{\partial t}=-k_{A F}^{0} \vec{B}+\vec{k}_{A F} \times \vec{E} .
$$

\footnotetext{
${ }^{11} \overline{\text { Note que os coeficientes } \tilde{\kappa}}$ são proporcionais ao quadrado do vetor $\vec{\beta}$, de forma que os correspondentes limites experimentais para $\vec{\beta}$ envolvem tomar a raiz quadrada. Limites experimentais da ordem $10^{-20}$ para certas componentes de $\tilde{\kappa}_{e-}$, por exemplo, obtidas por interferometria de lasers, implicariam em limites da ordem de $10^{-10}$ para o vetor $\vec{\beta}$.
}

Novamente, podemos investigar a propagação de ondas eletromagnéticas nesta teoria, e encontramos que a relação de dispersão é modificada da seguinte forma,

$$
\begin{aligned}
\omega^{2}-\vec{p}^{2} & = \pm\left(k_{A F}^{0}|\vec{p}|-\omega\left|\vec{k}_{A F}\right| \cos \theta\right) \\
& \times\left(1-\frac{\left|\vec{k}_{A F}\right|^{2} \sin ^{2} \theta}{\omega^{2}-\vec{p}^{2}}\right)^{-1 / 2},
\end{aligned}
$$

sendo $\theta$ o ângulo entre a propagação de onda e o vetor $\vec{k}_{A F}$. Novamente, vemos que a velocidade das ondas eletromagnéticas é diferente de $c=1 \mathrm{e}$, além disso, a presença do fator \pm indica a existência de duas polarizações com velocidades distintas, e isso vai levar novamente ao efeito de birrefringência, de forma similar ao que encontramos na subseção anterior.

Este efeito deve ser muito pequeno, então os autores tiveram a idéia de estudar a birrefringência num raio de luz que viaja uma distância $L$ muito grande. Para isso, procuraram dados de observações de galáxias, de tal modo que $L$ assumiria valores literalmente astronômicos. O problema é que, neste caso, não temos controle sobre as fontes desses raios de luz, então não podemos controlar a sua polarização inicial, apenas conseguimos medir a polarização da luz que chega ao detector, na Terra. Existem contudo casos em que temos uma idéia da polarização inicial da radiação: para galáxias e quasares que possuem uma forma alongada, percebe-se que a radiação emitida por essas fontes está polarizada preferencialmente nas direções paralelas e perpendiculares à direção da alongação. Essa correlação era conhecida na época, e havia modelos teóricos explicando esse fato. Os autores perceberam que a birrefringência induzida pela violação de Lorentz tenderia a girar o plano de polarização, "borrando" essa correlação. Com base nisso, coletaram os dados então disponíveis sobre a polarização da luz de mais de uma centena de fontes astronômicas distantes, e assumiram que os desvios com relação às direções preferenciais de polarização seriam devidas ao efeito de birrefringência do vácuo. Desta forma, conseguiram mostrar que as componentes de $k_{A F}$ deveriam ser extraordinariamente pequenas para serem compatíveis com as observações experimentais, da ordem de $10^{-42} \mathrm{GeV}$. Note que $k_{A F}$ possui dimensões de massa, então não podemos dizer que esse valor para $k_{A F}$ é "pequeno" de modo absoluto (esse tipo de afirmação só faz sentido para grandezas sem dimensão), contudo, essa escala de massa é extraordinariamente menor do que qualquer escala que aparece em teorias conhecidas, de forma que de fato podemos afirmar que $k_{A F}$ é em certo sentido "muito pequeno", e que os dados observacionais de galáxias distantes permitiram realizar um teste não trivial da simetria de Lorentz.

\section{Conclusões}

Neste trabalho, buscamos apresentar algumas das motivações para a busca por evidências experimentais de 
uma possível violação da simetria de Lorentz, bem como para o estudo sistemático de suas consequências teóricas. Inicialmente, fazendo um uso mínimo de equações, nos propomos a explicar em termos acessíveis o que essa frase significa, após, para o benefício de leitores com formação em física, apresentamos alguns modelos simples em que violações de Lorentz são incorporadas à eletrodinâmica. Mostramos como, a partir desses modelos, vínculos experimentais sobre possíveis violações de Lorentz podem ser encontrados.

A obtenção de uma teoria de gravitação quântica é um dos grandes problemas em aberto da física teórica, e a natureza mais fundamental do espaço-tempo está intimamente ligada a esse problema. Neste trabalho, argumentamos que os princípios fundamentais da Relatividade Restrita estão relacionados com a simetria do espaço-tempo (a simetria de Lorentz), e portanto tem uma conexão direta com esse célebre problema. Várias abordagens teóricas que pretendem capturar alguma faceta do que acontece na escala de Planck, domínio dessa elusiva teoria de gravitação quântica, prevêem de alguma forma pequenas violações da simetria de Lorentz. A possibilidade dessas violações é interessante porque envolve um domínio de fenômenos basicamente inexplorado, onde nova física muito naturalmente deve emergir, e pode ser vista como uma forma de testar a validade dos princípios da relatividade restrita nesse novo regime de fenômenos. Sendo a relatividade um dos pilares mais fundamentais da nossa compreensão teórica acerca do universo, do espaço-tempo, e das partículas que o habitam, estamos falando assim de testar os pilares mais fundamentais da física contemporânea.

Após delinear o cenário teórico que motiva o estudo de possíveis violações da simetria de Lorentz, descrevemos as idéias básicas que levam a um formalismo bastante geral, que estabelece uma linguagem comum tanto para os desenvolvimentos teóricos quanto para as buscas experimentais por violações de Lorentz, o chamado Modelo Padrão Extendido. Utilizamos o setor mais conhecido do Modelo Padrão, o setor eletromagnético, como protótipo para mostrar como essas análises são feitas, e comentamos sobre alguns dos resultados obtidos até aqui.

Até o momento, nenhuma evidência foi encontrada para a existência de violações de Lorentz, e por isso, as bases da Relatividade Restrita permanecem incólumes. Pode ser, afinal, que os postulados utilizados por Einstein no seu artigo de 1905 capturem uma verdade sobre a natureza, válida mesmo para seus regimes mais extremos - como as regiões próximas a um buraco negro, ou os primeiros instantes após o Big Bang. Esse seria um fato extraordinário, que portanto exigiria as mais extraordinárias evidências. Até o momento, o conjunto de limites experimentais organizado nas tabelas presentes em [30], resultado do esforço de uma comunidade de físicos teóricos e experimentais pelo menos desde a década de 1990 , tem se apresentado como tal evidência. Talvez, em algum momento do futuro, a validade da simetria de Lorentz na escala de Planck possa ser um dado experimental, e não uma suposição teórica - ou talvez algum futuro experimento nos revele surpresas!

\section{Agradecimentos}

$\mathrm{O}$ autor agradece à Fundação de Amparo à Pesquisa do Estado de São Paulo (FAPESP) e ao Conselho Nacional de Desenvolvimento Científico e Tecnológico (CNPq) pelo apoio financeiro, através dos projetos CNPq 304134/20171 e FAPESP 2017/13767-9; notadamente à FAPESP pelo apoio para realização de estágio de pesquisa no IUCSS - Indiana University Center for Spacetime Symmetries, durante a maior parte do ano de 2018. O autor agradece à hospitalidade do IUCSS durante esse estágio e, principalmente, ao Prof. V. A. Kostelecky, por muitas discussões sobre esta área de pesquisa. O autor também agradece a B. F. Braga, A. G. Dias e V. T. Zanchin pela leitura atenta do manuscrito e pelas várias correções sugeridas.

\section{Referências}

[1] A. Pais, Sutil é o Senhor: a ciência e a vida de Albert Einstein (Nova Fronteira, Rio de Janeiro, 1995).

[2] A.A.P. Vieira, Física na Escola 6, 83 (2005).

[3] L.C.B. Crispino e M.C. Lima, Rev. Bras. Ens. Fis. 40, 1601 (2017).

[4] D. Colladay e V.A. Kostelecky, Phys. Rev. D 55, 6760 (1997).

[5] D. Colladay e V.A. Kostelecky, Phys. Rev. D 58, 116002 (1998).

[6] P.A. Schulz, Rev. Bras. Ens. Fis. 29, 509 (2007).

[7] M. Novello, O que é cosmologia (Zahar, Rio de Janeiro, 2006).

[8] A.L.D. Fróes, Rev. Bras. Ens. Fis. 36, 3504 (2014).

[9] J.J.M. Pimenta, L.F.B Belussi, É.R.T. Natti e P.L. Natti, Rev. Bras. Ens. Fis. 35, 2306 (2013).

[10] M.A.M. Souza, A.C.S. Nascimento, D.F. Costa e O. Ferreira, Rev. Bras. Ens. Fis. 41, e20180124 (2019).

[11] M.C.B. Abdalla, O Discreto Charme das Partículas Elementares (Livraria da Física, São Paulo, 2006).

[12] B. Greene, O Universo Elegante (Companhia das Letras, São Paulo, 2001).

[13] J.T. Arantes, Cientistas simulam buraco negro em tanque de água, disponível em http://agencia.fapesp.br/cientistas-simulam-buraco-negro-em-tanque-deagua/29573/.

[14] R. Courtland, Physicists create 'black hole for sound', disponível em https://www.newscientist.com/article/dn17319-physicists-create-black-hole-for-sound/.

[15] D. Shiga, Hawking radiation glimpsed in artificial black hole, disponível em .

[16] D. Trancanelli, Rev. Bras. Ens. Fis. 38, 2505 (2016).

[17] R. Gambini e J. Pullin, A first course in Loop Quantum Gravity (Oxford University Press, Oxford, 2011).

[18] J. Ambjørn, J. Jurkiewicz e R. Loll, arXiv:1004.0352v1 (2010).

[19] A. Cho, Focus: Deriving Dimensions, disponível em . 
[20] A. Connes, Noncommutative Geometry (Academic Press, San Diego, 1994)

[21] B. Greene, O Tecido do Cosmo (Companhia das Letras, São Paulo, 2005).

[22] B. Lesche, Teoria da Relatividade (Livraria da Física, São Paulo, 2005).

[23] P.A. Tipler e R.A. Llewellyn, Física Moderna (LTC Editora, Rio de Janeiro, 2014).

[24] S.M. Carroll, J.A. Harvey, V.A. Kostelecky, C.D. Lane e T. Okamoto, Phys. Rev. Lett. 87, 141601 (2001).

[25] V.A. Kostelecky e S. Samuel, Phys. Rev. D 39, 683 (1989).

[26] H. Belich, T. Costa-Soares, M.A. Santos e M.T.D. Orlando, Rev. Bras. Ens. Fis. 29, 55 (2007).

[27] Q.G. Bailey e V.A. Kostelecky, Phys. Rev. D 70, 076006 (2004).

[28] R.G.G. Amorim, M.C.B. Fernandes, A.R. Queiroz, A.E. Santana e J.D.M. Viana, Rev. Bras. Ens. Fis. 35, 3604 (2013).

[29] T. Jacobson, S. Liberati e D. Mattingly, Annals Phys. 321, 150 (2006).

[30] V.A. Kostelecky e N. Russell, Rev. Mod. Phys. 83, 11 (2011).

[31] V.A. Kostelecky e M. Mewes, Phys. Rev. D 66, 056005 (2002).

[32] S.M. Carroll, G.B. Field e R. Jackiw, Phys. Rev. D 41, 1231 (1990).

[33] G.V. Dunne, arXiv:hep-th/9902115v1 (1999). 